\title{
Gentiana macrophylla exhibits a potential therapeutic effect on osteoarthritis (OA) via modulating disease- related proteins
}

\section{Type}

Research paper

\section{Keywords}

osteoarthritis, COX-2, Chondrocyte, PGE2, MMPs, Gentiana macrophylla

\begin{abstract}
Introduction

Prostaglandin E2 (PGE2) has been reported to cause cartilage degradation in the pathogenesis of osteoarthritis (OA). Matrix metallopeptidases (MMPs) play important roles in the pathogenesis of OA, while p-AKT and p-P39 signaling pathways were reported to be activated in the pathogenesis of OA. In this study, we aimed to investigate the effect of Gentiana macrophylla (GM) on the treatment of OA.

\section{Material and methods}

Primary rat chondrocytes were treated with PBS, IL-1 1 , and IL-1 $\beta+G M$ respectively to established in vitro models, and in vivo models were set up as a SHAM group, a monoiodoacetic acid (MIA) group, a MIA+GM (low dose) group and a MIA+GM (high dose) group.
\end{abstract}

\section{Results}

In primary rat chondrocytes, the IL-1 $\beta$ treatment elevated the expression of PGE2 and COX2 mRNA. However, the GM treatment reduced the expression of PGE2 mRNA and COX2 mRNA. Also, the GM treatment reduced the expression of above MMPs in primary rat chondrocytes treated with IL-1 $\beta$. Moreover, unlike P38 and AKT, GM treatment could reduce the expression of p-P38 and p-AKT in primary rat chondrocytes treated with IL-1 $\beta$. Also, GM treatment reduced the up-regulated expression of COX2, MMPs including MMP-1, MMP-3 and MMP-13, and p-P38 and p-AKT in OA rat models, thus exhibiting a therapeutic effect on OA pathology.

\section{Conclusions}

Our study demonstrated the inhibitory effect of GM on the up-regulated expression of PGE2, Cyclooxygenase-2 (COX-2), MMPs including MMP-1, MMP-3 and MMP-13, AKT and P38 in OA models, thus verifying the therapeutic effect of $G M$ on the treatment of $O A$. 

modulating disease-related proteins

3 Haitao Xu ${ }^{1}$, Ningyang Gao ${ }^{1}$, Yuxin Zheng ${ }^{1^{*}}$

1. Department of Orthopedics and Traumatology, Shuguang Hospital Affiliated to Shanghai University of Traditional Chinese Medicine, Shanghai 201203, China

* Correspondence to: Yuxin Zheng

Institution: Department of Orthopedics and Traumatology, Shuguang Hospital Affiliated to Shanghai University of Traditional Chinese Medicine

Address: No. 528 Zhangheng Road, Shanghai 201203, China

Email: oteoscience@163.com

\section{Abstract}

Background: Prostaglandin E2 (PGE2) has been reported to cause cartilage degradation in the pathogenesis of osteoarthritis (OA). Matrix metallopeptidases (MMPs) play important roles in the pathogenesis of OA, while p-AKT and p-P39 signaling pathways were reported to be activated in the pathogenesis of OA. In this study, we aimed to investigate the effect of Gentiana macrophylla (GM) on the treatment of OA. Method: Primary rat chondrocytes were treated with PBS, IL-1 $\beta$, and IL-1 $\beta+G M$ respectively to established in vitro models, and in vivo models were set up as a SHAM group, a monoiodoacetic acid (MIA) group, a MIA+GM (low dose) group and a MIA+GM (high dose) group. Results: In primary rat chondrocytes, the IL-1 $\beta$ treatment elevated the expression of PGE2 and COX2 mRNA. However, the GM treatment reduced the expression of PGE2 mRNA and COX2 mRNA. Also, the GM treatment reduced the expression of above MMPs in primary rat chondrocytes treated with IL-1ß. Moreover, unlike P38 and AKT, GM treatment could reduce the expression of p-P38 and p-AKT in primary rat chondrocytes treated with IL-1 3 . Also, GM treatment reduced the up-regulated expression of COX2, MMPs including MMP-1, MMP-3 and MMP-13, and p-P38 and p-AKT in OA rat models, thus exhibiting a therapeutic effect on OA pathology. Conclusion: Our study demonstrated the inhibitory effect of GM on the up- 
27 regulated expression of PGE2, Cyclooxygenase-2 (COX-2), MMPs including MMP-1, MMP-3 and MMP-13, AKT and P38 in OA models, thus verifying the therapeutic effect of GM on the treatment 29 of OA.

Running title: Gentiana macrophylla inhibits osteoarthritis by modulating the expression of 31 disease-related proteins

Keywords: Gentiana macrophylla, Osteoarthritis, PGE2, COX-2, MMPs, COX-2, chondrocyte

33

\section{Abbreviation}

OA: osteoarthritis

GM: Gentiana macrophylla

MIA: monoiodoacetic acid

\section{Introduction}

As a type of degenerative illness of the articular cartilage, osteoarthritis (OA), which is usually caused by the wear and tear of cartilage, impacts the normal life of roughly $1 / 3$ of the worldwide population [1]. Characterized by articular cartilage degradation and hypertrophic joint changes, the incidence of OA increases in older people. In China alone, more than half of the people with an age of $>65$ years old struggle with $O A$, which drastically influences their life quality [2]. OA is caused by numerous reasons, such as the aging of the worldwide population, joint stress, injury, as well as overweight, each one of which can lead to damages to the articular cartilage, triggering articular as well as subchondral bone hyperplasia. Thereby, OA causes symptoms including chronic joint inflammation, pain, as well as joint deformity [3-5].

GM is mainly used in China as well as Siberia. The root of GM contains some materials which are commonly utilized in Chinese medicine for the treatment of many health conditions, including diabetes, paralysis, apoplexy, as well as rheumatism [6]. GM belongs to the Gentianaceae family. The dried root of GM has been widely used as a Chinese medicinal herb. The active compounds of GM, including gentiopicroside, loganic acid, swertiamarin and sweroside, are enriched in the plant and exert extensive pharmacological effects, including choleretic, stomachic, anti- 
inflammatory, anti-hepatotoxic, as well as also antihistamine effects $[7,8]$. Therefore, GM is often made use of to handle rheumatoid arthritis [7]. Moreover, GM has been reported to be useful in the management of apoplexy, diabetes, liver injuries, and rheumatism [9-11]. Especially, GM has been demonstrated to be a useful anti-inflammatory agent in the control of inflammation of rheumatoid arthritis [11].

MMPs belong to the family of proteolytic enzymes dependent on zinc and play a role in protein degradation [12]. MMPs may degrade any kind of extracellular matrix component, such as vitronectin, proteoglycans, laminin, fibronectin, as well as collagens. From a standpoint of disease progression, MMPs are very important during tumor growth as well as metastasis [13, 14]. For that reason, the levels of MMP-1, MMP-9 as well as MMP-2 proteins in OA patients were increased to promote the progress of tumors and illnesses such as OA. Among MMPs, attention has been drawn to MMP-13 considering that it is substantially overexpressed in the articular cartilage as well as joint tissues in OA patients but is lowly expressed in normal tissues. In a mouse model of OA, the level of MMP-13 was associated with the chondrocytes collected from OA rats during the onset of OA [15]. The over-expression of MMP-13 can trigger the onset of OA via excessive degradation of the extracellular matrix (ECM) $[16,17]$.

The family of p38 mitogen-activated protein kinases (MAPK) is made up of 4 proteins: p38 $\alpha, p 38 \beta$, $p 38 \gamma$, as well as $p 38 \delta$. Among these kinases which are expressed in a tissue-specific manner, p38 $\alpha$ is ubiquitously expressed at significant levels in most cell types [18]. Besides, p38 MAPKs act as substrates of 3 MAP2K enzymes, i.e., MKK6, MKK4 as well as MKK3). The treatment with inhibitors of p38 MAPK prevents inflammation as well as joint degradation in mice, showing the potential of using p38 MAPK inhibitors in the treatment of OA [19]. Past studies also revealed that the CAMP response element-binding protein (CREB) activation led to boosted MMP13 expression in chondrocytes $[20,21]$. In addition, the activation of CREB induced by IL-1 $\beta$ is mostly depending on the action of $p 38$ kinases $[22,23]$. Thus, the expression of $p-P 38$, as well as $p-C R E B$, was elevated in osteoarthritic cartilages [24].

It has been shown that IL-18 may induce the production of PGE2, which led to cartilage degradation in the pathogenesis of OA [25]. Meanwhile, celecoxib could diminish the levels of 
COX-2 and promote the degeneration of cartilage in the progression of OA and bone healing [26]. Moreover, MMPs including MMP-1, MMP-3 and MMP-13 were reported to play important roles in the pathogenesis of OA [15-17]. It was also reported that the down-regulated p-AKT in the cartilage tissue could promote chondrocyte apoptosis and autophagy in the pathogenesis of OA [27]. And the p-P38 signaling pathway up-regulated the expression of iNOS and COX-2, which induced apoptosis in the pathogenesis of OA [28]. In this study, we hypothesized that GM might exhibit its therapeutic effect upon OA via modulating the expression of the above-mentioned genes and proteins. To validate our hypothesis, we established cellular and animal models and administrated GM to observe the effect of GM during the treatment of OA via observing its effect on the expression of PGE2, COX-2, AKT, P38 and MMPs including MMP-1, MMP-3 and MMP-13.

\section{Materials and Methods}

\section{Extract preparation}

The extract preparation was accomplished following instructions given by a previous publication [9]. Briefly, we utilized Gentiana macrophylla roots which were harvested in autumn and dried naturally under the sun (Gansu Provincial Medical Company, Gansu, China). $500 \mathrm{~g}$ of the filtered fine powder (by 40 mesh filter) of the roots of GM (harvested from field) were treated with $70 \%$ ethanol for $1 \mathrm{~h}$ under reflux, and the extraction was repeated twice and the extract from both extractions was pooled. In the next step, the extract was concentrated via running through a gel column packed with molecular adsorption silica (200-400 mesh) and then eluted by using chloroform. Then, the mixture of the extract of GM and chloroform was dried and then ground to produce a brownish powder. During use, the powder was reconstituted using distilled water to produce solutions at desired concentrations.

\section{Animal model and treatment}

A total of 28 Sprague-Dawley Male Rats (with an average age of 3 months old and an average bodyweight of $250 \mathrm{~g}$ ) were utilized in this study. All SD rats were placed in animal cages with each cage holding five rats. In addition, the conditions of the animal room complied with the requirements of the SPF grade. During the study, all rats were given unlimited access to water and food, and the environment in the animal room was set to a humidity level of $50 \%$ and a 
constant temperature of $22^{\circ} \mathrm{C}$. The animal model was established according to a previous publication [29]. Briefly, during the experiments, the rats were divided randomly into 4 groups with 4 rats in each group: 1. SHAM group (SD rats treated with sterile saline); 2 . MIA group (SD rats treated with MIA to induce OA; 3. MIA + GM group-low dose (SD rats treated with MIA to induce $\mathrm{OA}$ and at the same time given a $100 \mathrm{mg} / \mathrm{kg}$ body weight of GM); 4. MIA + GM group-high dose (SD rats treated with MIA to induce OA and at the same time given a $200 \mathrm{mg} / \mathrm{kg}$ body weight of GM). All rats in the MIA groups were given MIA through intra-articular injection via the infrapatella ligament in the right knee, and the dose of MIA was $50 \mu$ of sterile saline containing $1 \mathrm{mg}$ of MIA for each rat. During the injection of MIA, all rats were anesthetized intraperitoneally with ketamine $(75 \mathrm{mg} / \mathrm{kg})+$ dexmedetomidine $(0.5 \mathrm{mg} / \mathrm{kg})$. The rats in the SHAM group were given the intra-articular injection of sterile saline in the same volume. In terms of administration of GM, the rats in the two MIA + GM groups were given the extract of GM, which was obtained via the method described above, via oral administration at a fixed dose of one hundred milligrams per one kilogram of body weight. The cartilage tissues of the rat hip joints were collected for subsequent analysis. At the end of the study, the rats were sacrificed by a lethal intravenous dose of sodium pentobarbital $(100 \mathrm{mg} / \mathrm{kg})$ in strict compliance with the "Guide for the Care and Use of Laboratory Animals" published by the US National Institutes of Health (NIH). All experimental operations involving animal treatment were carried out upon approval from the Animal Ethics Committee of our university.

\section{Isolation of primary rat chondrocytes}

After SD rats were adapted to the conditions of the SPF environment, primary chondrocytes were collected from the hip joints of the rats. In brief, the cartilage tissues of the rats were collected from their hip joints and then minced into $1 \mathrm{~mm} 3$ cubes under a sterile environment. After that, the cartilage tissues were trypsinized for $1 \mathrm{~h}$ at room temperature with $0.25 \%$ trypsin before they were incubated for $6 \mathrm{~h}$ at $37^{\circ} \mathrm{C}$ and under 5\% carbon dioxide in DMEM-F12 (Gibco, Thermo Fisher Scientific, Waltham, MA) containing $0.2 \%$ collagenase II. Afterward, the suspension of chondrocytes was centrifuged for 5 minutes at $241.5 \mathrm{xg}$ and then cultured in DMEM-F12 containing $10 \%$ FBS as well as $1 \%$ suitable antibiotics. 


\section{Cell culture and treatment}

Chondrocytes were harvested from SD rats and then maintained in a special growth medium of chondrocytes in an environment of $5 \% \mathrm{CO}_{2}$ and $37{ }^{\circ} \mathrm{C}$ under saturated humidity. Then, cellular models were established according to a previous publication [30]. Briefly, the cells were divided into 3 groups, i.e., 1. PBS group (rat chondrocytes treated with PBS only); 2. IL-1 $\beta$ group (rat

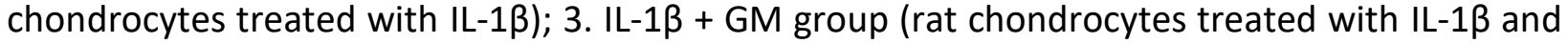
$\mathrm{GM})$. In the IL-1 $\beta$ groups, the growth medium was replaced by a medium containing no serum

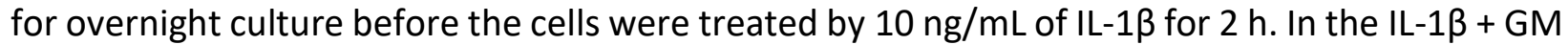
group, the cells were treated with $10 \mathrm{ng} / \mathrm{mL}$ of IL-1 $\beta$ along with $5 \mu \mathrm{M}$ of GM for $2 \mathrm{~h}$. Subsequently, the cells were harvested for real-time PCR and Western blot to measure the expression of target genes.

\section{RNA isolation and real-time PCR}

The isolation of total RNA from tissue and cell samples was carried out by using a Trizol reagent (Invitrogen, Carlsbad, CA) based on the instruction manual provided by the assay kit manufacturer. To determine the expression of COX2 mRNA, MMP-1 mRNA, MMP-3 mRNA, and MMP-13 mRNA in the samples, the extracted total RNA in each sample was first reverse transcribed into cDNA by using an iScript cDNA Synthesis assay kit (Bio-Rad Laboratories, Hercules, CA) based on the instruction manual provided by the assay kit manufacturer. In the next step, real-time PCR was done by utilizing an iQ SYBR Green Supermix (Bio-Rad Laboratories, Hercules, CA) based on the instruction manual provided by the assay kit manufacturer, on a 7900HT real-time PCR machine (Applied Biosystems, Foster City, CA). Finally, the relative expression of COX2 mRNA, MMP-1 mRNA, MMP-3 mRNA, and MMP-13 mRNA was calculated using the 2 delta delta Ct method. GAPDH was used as the internal reference.

\section{Western blot analysis}

To determine the protein expression of COX2, MMP-1, MMP-3, MMP-13, P38, p-P38, AKT, and p-AKT in each sample, the tissue and cell samples were first lysed in a RIPA buffer (Invitrogen, Carlsbad, CA) based on the instruction manual provided by the reagent manufacturer. In the next step, the isolated protein from each sample $(50 \mu \mathrm{g})$ was resolved by using $10 \%$ SDS-PAGE and 
blotted onto PVDF membranes, which were then blocked for $2 \mathrm{~h}$ at room temperature with $5 \%$ non-fat milk and then treated with primary antibodies against COX2 (Dilution 1:1000; ab179800; Abcam, Cambridge, MA), MMP-1 (Dilution 1:1000; ab134184; Abcam, Cambridge, MA), MMP-3 (Dilution 1:1000; ab52915; Abcam, Cambridge, MA), MMP-13 (Dilution 1:1000; ab51072; Abcam, Cambridge, MA), P38 (Dilution 1:1000; \# 9212S; Cell Signaling Technology, Danvers, MA), p-P38 (Dilution 1:1000; \#9211S; Cell Signaling Technology, Danvers, MA), AKT (Dilution 1:500; \# 9272S; Cell Signaling Technology, Danvers, MA), and p-AKT (Dilution 1:1000; \# 9271S; Cell Signaling Technology, Danvers, MA) based on the suggested conditions of antibody incubation provided by the antibody manufacturer. Subsequently, the PVDF membranes were washed and further incubated with HRP-labeled secondary antibodies for $1 \mathrm{~h}$ at room temperature (Beyotime, Shanghai, China). Finally, the protein bands were developed by using an enhanced chemiluminescence $(E C L)$ assay kit (Millipore, Billerica, $M A$ ) based on the instruction manual provided by the assay kit manufacturer and then visualized to determine the relative expression of COX2, MMP-1, MMP-3, MMP-13, P38, p-P38, AKT, and p-AKT proteins in each sample. The protein quantification was performed by optic density analysis of each protein band by Image J software (Version no. 1.44e, National Institutes of Health). $\beta$-actin was used as the control.

\section{ELISA}

The expression of PGE2 in collected samples was determined by using standard commercial enzyme-linked immunosorbent assay (ELISA) kits (Thermo Fisher Scientific, Waltham, MA) based on the instruction manual provided by the assay kit manufacturer.

\section{IHC assay}

To determine the protein expression of p-P38 and p-AKYT in the rat cartilage tissue samples collected from the hip joints, the samples were first fixed in $4 \%$ paraformaldehyde (Sigma Aldrich, St Louis, MO), embedded in paraffin, sliced into $5 \mu \mathrm{m}$ sections, stained with anti-p-P38 and antip-AKYT primary and biotinylated secondary antibodies (Abcam, Cambridge, MA), and counterstained with hematoxylin before the positive expression of p-P38 and p-AKYT proteins was analyzed under an Olympus light microscope (magnification, x200; Olympus Corporation) by semi-quantitative analysis. 
All statistical analyses were carried out by using SPSS 21.0 (IBM, Chicago, IL). The differences between different groups were compared by using one-way ANOVA. A P value of $<0.05$ was deemed statistically significant. Each experiment was repeated in triplicate.

\section{Results}

PGE2 production and COX-2 mRNA expression were elevated by IL-1 $\beta$ treatment and inhibited by GM treatment

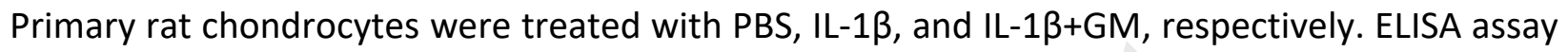
was performed to compare the production of PGE2 among different cell groups. As shown in Fig.1A, the PGE2 production was evidently increased in the IL-1 $\beta$ treatment group while GM treatment inhibited the expression of PGE2. Also, the relative gene expression of COX-2 (Fig.1B) was increased by the IL-1 $1 \beta$ treatment and was reduced by the co-treatment of GM. Therefore, it was validated that IL-1 $\beta$ treatment elevated the expression of PGE2 MRNA and COX2 MRNA in primary rat chondrocytes, while the GM treatment reduced the gene expression of PGE2 mRNA and COX2 mRNA to a certain degree.

MMP expression was elevated by IL-1 $\beta$ and inhibited by GM treatment

Moreover, real time qPCR results showed that the IL-1 $\beta$ treatment caused a significant elevation in the relative gene expression of MMPs, including MMP-1 (Fig.2A), MMP-3 (Fig.2B) and MMP13 (Fig.2C), in primary rat chondrocytes, and the gene expression of MMPs was suppressed by

212 the co-treatment with GM. Moreover, the protein expression of MMPs, including MMP-1 (Fig.3A), 213 MMP-3 (Fig.3B) and MMP-13 (Fig.3C), was also increased in the IL-1 $\beta$ group, and GM treatment 214 reduced the protein expression of these MMPs. Therefore, it was validated that IL-1 $\beta$ treatment 215 elevated the expression of MMPs, including MMP-1, MMP-3 and MMP-13 in primary rat 216 chondrocytes, while the GM treatment reduced the expression of these MMPs to a certain 217 degree. 
The production of P38, p-P38, AKT and p-AKT was measured by Western blot assays. Accordingly, the levels of P38 (Fig.4A) and AKT (Fig.4C) remained similar among different groups, while the levels of p-P38 (Fig.4C) and p-AKT (Fig.4D) were both increased by the treatment of IL-1 3 . Moreover, the up-regulated production of p-P38 and p-AKT in the IL-1 13 group was both downregulated by GM treatment. Therefore, it was validated that the GM treatment partly downregulated the expression of p-P38 and p-AKT elevated in the primary rat chondrocytes treated with IL-1ß.

\section{GM treatment inhibited the up-regulated COX2 and MMPs in OA rats}

Four groups of rats were established in our study: a SHAM group, an MIA group, a MIA+GM (low dose) group and a MIA+GM (high dose) group. Keen articular cartilage tissues were collected and subjected to PCR and Western blot assays to measure the expression of COX2 mRNA and MMPs. As shown in Fig.5, the relative expression levels of COX2 mRNA (Fig.5A), MMP-1 mRNA (Fig.5B), MMP-3 mRNA (Fig.5C) and MMP-13 mRNA (Fig.5D) were all significantly increased in OA rats (MIA group), and GM treatment suppressed the up-regulated gene expression of COX2 and MMPs in a dose-dependent manner. Moreover, the relative protein expression of COX2 (Fig.6A), MMP-1 (Fig.6B), MMP-3 (Fig.6C) and MMP-13 (Fig.6D) was all markedly up-regulated in OA rats. A low dose of GM treatment alleviated the up-regulated COX2 and MMPs in the MIA group, while a high dose of GM treatment exhibited a more significant inhibitory effect on COX2 and MMPs in the MIA group. Therefore, it was concluded that GM treatment alleviated the up-regulated COX2, MMP-1, MMP-3 and MMP-13 in OA rats, thus exhibiting a therapeutic effect on OA pathology.

\section{GM treatment inhibited the up-regulated p-P38 and p-AKT in OA rats}

Furthermore, the production of P38, p-P38, AKT and p-AKT was observed in rats via Western blot and IHC assays. As shown in Fig.7, the Western blot assay exhibited no evident difference of P38 production (Fig.7A) or AKT expression (Fig.7C) among the rat groups, while the production of pP38 (Fig.7B) and p-AKT (Fig.7D) was significantly increased in OA rats, but GM treatment reduced the p-P38 and p-AKT production in OA rats. Similarly, the results from IHC assays also indicated the inhibitory effect of GM on the p-P38 (Fig.8A) and p-AKT (Fig.8B) production evidently upregulated in OA rats. 
248

249

250

251

252

253

254

255

256

257

258

259

260

261

262

263

264

265

266

267

268

269

270

271

272

273

274

In this study, we found that GM treatment of OA rats alleviated the up-regulated expression of COX2, MMP-1, MMP-3 and MMP-13, thus exhibiting a therapeutic effect on OA pathology. In addition, GM treatment inhibited the up-regulated p-P38 and p-AKT expression in OA rats. The results of this present study are consistent with previous studies about the therapeutic role of GM in the treatment of various human medical conditions. For instance, it was found that iridoid glycosides from G. macrophylla (GMI) could remarkably reduce the levels of COX-2 as well as iNOS. It was speculated that COX-2 as well as iNOS signaling is involved in the GM activity in RA treatment [31]. Another research showed that the treatment of OA with COX-2 inhibitors as well as metformin could lower the rates of joint replacement surgery [32]. The different therapeutic effect of GM on different diseases may be attributed to its effects on different disease-related genes or signaling pathways and its effect on OA involves COX2, MMP-1, MMP-3 and MMP-13.

IL-1 $\beta$ activates COX-2 expression and PGE2 production in chondrocytes [33]. In addition, the expression of COX- 2 proteins is often delayed after the activation of chondrocytes by IL-1 $\beta$ in OA [34]. It was shown that the level of PGE2 was more effectively decreased by GM than by prednisone. When an extract of GM rats was administered into rats, the level of PGE2 was obviously decreased to reduce chondrocyte damage [9]. Since the increase in PGE2 can result in inflammatory responses in RA, including edema as well as pain, the significantly decreased PGE2 level in rat models of RA may be a result from COX2 inhibition by GM [35]. It was revealed that exogenous administration of PGE2 in chondrocytes of rats induced the incorporation of [3H] thymidine as well as the synthesis of aggrecan which was reported to be involved in the pathogenesis of OA [36]. In human cartilages affected by OA, exogenous administration of PGE2 up-regulated the levels of glucocorticoid receptors, suggesting a possible negative feedback loop of PGE2 $[37,38]$. All those above previous reports indicate a pivotal role of COX2/PEG2 in the development of $\mathrm{OA}$ and the inhibitory effect of GM on this signaling pathway might be the major reason for its therapeutic effect of GM in OA.

It was shown that MMP-13 was expressed abnormally in human cartilages in different stages of OA [39]. Due to the fact that MMP-13 plays a core role in the degradation of cartilage, the 
275 inhibition of expression of MMP-13 has been thought to be an effective way to limit the development of $\mathrm{OA}$ and the regulation of MMP-13 expression could be moderated at different levels, such as post-transcriptional regulation, epigenetic regulation, as well as transcriptional regulation [40-42]. The result of this study indicated that GM treatment down-regulated expression of MMP-13 but how GM regulated the expression of MMP-13 is still under investigation. In addition, MMP-13 is suggested to contribute to the turnover of cartilage materials via cleaving ECM molecules including type II collagen $[43,44]$. MMP-13 is constitutively generated and endocytosed by chondrocytes [41]. During the progression of OA, the induction of collagenase as well as MMP-13 activity was noticed in cartilages [45-47]. In this study, we found that IL-1 $\beta$ treatment elevated the expression of PGE2 and COX2 mRNAs in primary rat chondrocytes. However, the GM treatment reduced the gene expression of PGE2 mRNA and COX2 mRNA. In addition, we also found that IL-1 $\beta$ treatment elevated the expression of MMPs, including MMP-1, MMP-3 and MMP-13, in primary rat chondrocytes, but the GM treatment reduced the expression of these MMPs elevated by IL-1 $1 \beta$. Meanwhile, we also found that GM treatment reduced the expression of $\mathrm{p}-\mathrm{P} 38$ and $\mathrm{p}-\mathrm{AKT}$ elevated by IL-1 $\beta$ in primary rat chondrocytes.

MAPK signaling, such as JNK as well as p38 MAPK pathways, were suggested to be widely associated with the progression of OA $[48,49]$. Thereby, the p38 MAPK activation is associated with chondrocyte apoptosis, while the p38 MAPK inhibition is associated with reduced cartilage damage in OA [50]. A past study actually illustrated that AKT controls the proliferation of chondrocytes during skeletal development $[51,52]$. Thus, the inhibition of the PI3K/ AKT/mTOR pathway might play a role in preventing chondrocyte expansion.

\section{Conclusion}

Our study demonstrated the inhibitory effect of GM on the expression of PGE2, COX-2, MMPs including MMP-1, MMP-3 and MMP-13, AKT and P38 up-regulated in IL-1ß cell group or MIA rat group, thus verifying the therapeutic effect of GM on the treatment of OA.

\section{Conflict of interest}

None 
304 This work was supported by Shanghai Sailing Program (19YF1449700).

Availability of data and material

The data that support the findings of this study are available from the corresponding author upon

307 reasonable request.

\section{Authors' contributions}

YXZ and HTX planned the study, HTX and NYG collected the literature,collected and analyzed the

310 data, YXZ and NYG composed the manuscript, and all the other co-authors approved the final 311 manuscript.

\section{Figure legends}

\section{$313 \quad$ Fig.1}

314 Expression of PGE2 and COX-2 mRNAs elevated by the IL-1 $\beta$ treatment was inhibited by the GM 315 treatment $(\mathrm{N}=3 ;$ * $\mathrm{P}$ value $<0.05$ vs. PBS group; ** $\mathrm{P}$ value < 0.05 vs. IL-1 $\beta$ group; one-way ANOVA)

316 A: ELISA assay indicated that PGE2 production was increased in primary rat chondrocytes treated 317 with IL-1 $\beta$, while GM treatment inhibited the increased PGE2 production in IL-1 $\beta$ group;

318 B: Real time PRC indicated that the relative expression of COX2 mRNA was increased in primary 319 rat chondrocyte $s$ treated with IL-1 $\beta$, while GM treatment inhibited the increased PGE2 320 production in IL-1ß group.

$321 \quad$ Fig. 2

322 The expression of MMPs elevated by the IL-1 $\beta$ treatment was inhibited by the GM treatment (N3233 ; * P value < 0.05 vs. PBS group; ** P value < 0.05 vs. IL-1 $\beta$ group; one-way ANOVA)

324 A: Real time PRC indicated that the relative expression of MMP-1 mRNA was increased in primary 325 rat chondrocyte $s$ treated with IL-1 $\beta$, while GM treatment inhibited the increased MMP-1 mRNA 326 level in IL-1 $\beta$ group; 
B: Real time PRC indicated that the relative expression of MMP-3 mRNA was increased in primary rat chondrocyte s treated with IL-1 $\beta$, while GM treatment inhibited the increased MMP-3 mRNA 329 level in IL-1 $\beta$ group;

C: Real time PRC indicated that the relative expression of MMP-13 mRNA was increased in primary rat chondrocyte s treated with IL-1 $\beta$, while GM treatment inhibited the increased MMP13 mRNA level in IL-1 $\beta$ group.

Fig.3

334 Protein expression of MMPs elevated by the IL-1 $\beta$ treatment was inhibited by the GM treatment $335\left(\mathrm{~N}=3 ; * \mathrm{P}\right.$ value $<0.05$ vs. PBS group; ${ }^{* *} \mathrm{P}$ value $<0.05$ vs. IL-1 $\beta$ group; one-way ANOVA)

A: Western blot assay indicated that the protein level of MMP-1 was up-regulated in primary rat chondrocyte s treated with IL-1 $\beta$, while GM treatment suppressed the up-regulation of MMP-1 protein in IL-1 $\beta$ group;

B: Western blot assay indicated that the protein level of MMP-3 was up-regulated in primary rat chondrocyte s treated with IL-1 $\beta$, while GM treatment suppressed the up-regulation of MMP-3

341 protein in IL-1 $\beta$ group;

342 C: Western blot assay indicated that the protein level of MMP-13 was up-regulated in primary 343 rat chondrocyte s treated with IL-1 $\beta$, while GM treatment suppressed the up-regulation of MMP34413 protein in IL-1 $\beta$ group.

Fig.4

346 The protein production of p-P38 and p-AKT elevated by the IL-1 $\beta$ treatment was inhibited by the 347 GM treatment ( $\mathrm{N}=3$; * $\mathrm{P}$ value < 0.05 vs. PBS group; ** $\mathrm{P}$ value < 0.05 vs. IL-1 $\beta$ group; one-way 348 ANOVA)

349 A: Western blot assay indicated that the protein levels of P38 remained stable among different 350 primary rat chondrocyte groups;

351 B: Western blot assay indicated that the up-regulated protein level of p-P38 in primary rat 352 chondrocytes treated with IL-1 $\beta$ was evidently reduced by the administration of GM; 
C: Western blot assay indicated that the protein levels of AKT remained stable among different primary rat chondrocyte groups;

D: Western blot assay indicated that the up-regulated protein level of p-AKT in primary rat chondrocytes treated with IL-1 $\beta$ was evidently reduced by the administration of GM.

\section{$357 \quad$ Fig.5}

358 GM treatment inhibited the gene expression of COX2 and MMPs up-regulated in OA rats $(\mathrm{N}=3$; *

359 P value $<0.05$ vs. sham group; ** P value < 0.05 vs. MIA group; one-way ANOVA)

A: Real time PCR indicated that the relative gene expression of COX2 was significantly increased in MIA group, while being partly restored in MIA+GM group in a dose-dependent manner;

B: Real time PCR indicated that the relative gene expression of MMP-1 was significantly increased in MIA group, while being partly restored in MIA+GM group in a dose-dependent manner;

C: Real time PCR indicated that the relative gene expression of MMP-3 was significantly increased in MIA group, while being partly restored in MIA+GM group in a dose-dependent manner;

D: Real time PCR indicated that the relative gene expression of MMP-13 was significantly increased in MIA group, while being partly restored in MIA+GM group in a dose-dependent 368 manner.

$369 \quad$ Fig.6

$370 \mathrm{GM}$ treatment inhibited the protein production of COX2 and MMPs up-regulated in OA rats $(\mathrm{N}=3$;

$371 * \mathrm{P}$ value < 0.05 vs. sham group; ** P value < 0.05 vs. MIA group; one-way ANOVA)

372 A: Western blot assay indicated that the relative protein expression of COX2 was significantly 373 increased in MIA group, while being partly restored in MIA+GM group in a dose-dependent 374 manner;

375 B: Western blot assay indicated that the relative protein expression of MMP-1 was significantly 376 increased in MIA group, while being partly restored in MIA+GM group in a dose-dependent 377 manner; 
C: Western blot assay indicated that the relative protein expression of MMP-3 was significantly increased in MIA group, while being partly restored in MIA+GM group in a dose-dependent manner;

D: Western blot assay indicated that the relative protein expression of MMP-13 was significantly increased in MIA group, while being partly restored in MIA+GM group in a dose-dependent manner.

\section{Fig.7}

The Western blot assay showed that the GM treatment inhibited the p-P38 and p-AKT production 386 which were up-regulated in $\mathrm{OA}$ rats $(\mathrm{N}=3 ; * \mathrm{P}$ value $<0.05$ vs. sham group; $* * \mathrm{P}$ value $<0.05$ vs. 387 MIA group; one-way ANOVA)

388 A: Western blot assay indicated that the relative protein expression of P38 remained stable 389 among different rat groups;

390

B: Western blot assay indicated that the relative protein expression of p-P38 was evidently 391 promoted in MIA group, and GM treatment partly restored the p-P38 up-regulation in MIA+GM 392 group in a dose-dependent manner.

393 C: Western blot assay indicated that the relative protein expression of AKT remained stable 394 among different rat groups;

395 396 397 group in a dose-dependent manner.

$398 \quad$ Fig.8

399 IHC assay showed that GM treatment inhibited the p-P38 and p-AKT production which were up400 regulated in OA rats (Scale bar: $50 \mu \mathrm{m}$ )

401 A: IHC assay demonstrated inhibitory effect of GM upon the up-regulated expression of p-P38 in 402 the MIA rats treated with different doses of GM; 
B: IHC assay demonstrated inhibitory effect of GM upon the up-regulated expression of $p$-AKT in

404 the MIA rats treated with different doses of GM.

405

4061

407

408

409

410

411

412

413

414

415

4164

417

418

419

$420 \quad 5$

421

422

$423 \quad 6$

424

425

$426 \quad 7$

427

428

429

430

\section{References}

1 Rahimzadeh P, Imani F, Faiz SH, Entezary SR, Nasiri AA, Ziaeefard M: Investigation the efficacy of intra-articular prolotherapy with erythropoietin and dextrose and intraarticular pulsed radiofrequency on pain level reduction and range of motion improvement in primary osteoarthritis of knee. J Res Med Sci 2014;19:696-702.

2 Gronhaug G, Osteras N, Hagen KB: Quality of hip and knee osteoarthritis management in primary health care in a Norwegian county: a cross-sectional survey. BMC Health Serv Res 2014;14:598.

3 Bao JP, Jiang LF, Chen WP, Hu PF, Wu LD: Expression of vaspin in the joint and the levels in the serum and synovial fluid of patients with osteoarthritis. Int J Clin Exp Med 2014;7:3447-3453.

4 Sowa GA, Perera S, Bechara B, Agarwal V, Boardman J, Huang W, Camacho-Soto A, Vo N, Kang J, Weiner D: Associations between serum biomarkers and pain and pain-related function in older adults with low back pain: a pilot study. J Am Geriatr Soc 2014;62:20472055.

5 Yang Y, Tien HY, Kumar KK, Chen S, Li Z, Tian W, Tian G: Ligament reconstruction with tendon interposition arthroplasty for first carpometacarpal joint osteoarthritis. Chin Med J (Engl) 2014;127:3921-3925.

$6 \quad$ Hu YZ, Wei JY, Tang SH, Yang HJ: Analysis on composition principles of formulae containing Gardeniae Fructus in dictionary of traditional Chinese medicine prescriptions. Zhongguo Zhong Yao Za Zhi 2016;41:1342-1347.

7 Wang Y, Ahmad B, Duan B, Zeng R, Huang L: Chemical and Genetic Comparative Analysis of Gentiana crassicaulis and Gentiana macrophylla. Chem Biodivers 2016;13:776-781

8 Jiang ZB, Liu HL, Liu XQ, Shang JN, Zhao JR, Yuan CS: Chemical constituents of Gentiana macrophylla Pall. Nat Prod Res 2010;24:1365-1369. 
4319 Chen LY, Xu ZQ: Somatic embryogenesis pathway for plant regeneration in Qinjiao 432 (Gentiana macrophylla Pall.). Fen Zi Xi Bao Sheng Wu Xue Bao 2007;40(4):267-71.

43310 Sheu MJ, Chiu CC, Yang DJ, Hsu TC, Tzang BS. The Root Extract of Gentiana macrophylla 434 Pall. Alleviates B19-NS1-Exacerbated Liver Injuries in NZB/W F1 Mice. J Med Food $435 \quad 2017 ; 20(1): 56-64$.

43611 Yu F, Yu F, Li R, Wang R: Inhibitory effects of the Gentiana macrophylla (Gentianaceae) 437 extract on rheumatoid arthritis of rats. J Ethnopharmacol 2004;95:77-81.

43812 Bourboulia D, Stetler-Stevenson WG: Matrix metalloproteinases (MMPs) and tissue 439 inhibitors of metalloproteinases (TIMPs): Positive and negative regulators in tumor cell 440 adhesion. Semin Cancer Biol 2010;20:161-168.

442

13 Salgame P: MMPs in tuberculosis: granuloma creators and tissue destroyers. J Clin Invest

44314 Lukaszewicz-Zajac M, Mroczko B, Kornhuber J, Lewczuk P: Matrix metalloproteinases 444

15 Kamekura S, Hoshi K, Shimoaka T, Chung U, Chikuda H, Yamada T, Uchida M, Ogata N, 447 (MMPs) and their tissue inhibitors (TIMPs) in the tumors of central nervous system (CNS). J Neural Transm (Vienna) 2014;121:469-477. Seichi A, Nakamura K, Kawaguchi H: Osteoarthritis development in novel experimental mouse models induced by knee joint instability. Osteoarthritis Cartilage 2005;13:632-641.

18 Cuadrado A, Nebreda AR: Mechanisms and functions of p38 MAPK signalling. Biochem J 2010;429:403-417.

45719 Maudens P, Seemayer CA, Pfefferle F, Jordan O, Allemann E: Nanocrystals of a potent p38 MAPK inhibitor embedded in microparticles: Therapeutic effects in inflammatory and mechanistic murine models of osteoarthritis. J Control Release 2018;276:102-112. 
46020 Ha YJ, Choi YS, Kang EH, Shin K, Kim TK, Song YW, Lee YJ: SOCS1 suppresses IL-1ßetainduced C/EBPbeta expression via transcriptional regulation in human chondrocytes. Exp Mol Med 2016;48:e241.

46321 Bui C, Barter MJ, Scott JL, Xu Y, Galler M, Reynard LN, Rowan AD, Young DA: cAMP response element-binding (CREB) recruitment following a specific CpG demethylation 466

22 Frost RA, Nystrom GJ, Lang CH: Stimulation of insulin-like growth factor binding protein468 1 synthesis by interleukin-1beta: requirement of the mitogen-activated protein kinase pathway. Endocrinology 2000;141:3156-3164.

23 Funding AT, Johansen C, Kragballe K, Iversen L: Mitogen- and stress-activated protein kinase 2 and cyclic AMP response element binding protein are activated in lesional psoriatic epidermis. J Invest Dermatol 2007;127:2012-2019.

24 Ji B, Ma Y, Wang H, Fang X, Shi P: Activation of the P38/CREB/MMP13 axis is associated with osteoarthritis. Drug Des Devel Ther 2019;13:2195-2204.

47525 Futani H, Okayama A, Matsui K, Kashiwamura S, Sasaki T, Hada T, Nakanishi K, Tateishi H, Maruo S, Okamura H: Relation between interleukin-18 and PGE2 in synovial fluid of osteoarthritis: a potential therapeutic target of cartilage degradation. J Immunother

47926 Mastbergen SC, Marijnissen AC, Vianen ME, Zoer B, van Roermund PM, Bijlsma JW, 480 Lafeber FP: Inhibition of COX-2 by celecoxib in the canine groove model of osteoarthritis. Rheumatology (Oxford) 2006;45:405-413.

28 Pelletier JP, Fernandes JC, Jovanovic DV, Reboul P, Martel-Pelletier J: Chondrocyte death 486 in experimental osteoarthritis is mediated by MEK $1 / 2$ and p38 pathways: role of cyclooxygenase-2 and inducible nitric oxide synthase. J Rheumatol 2001;28:2509-2519. 
29 Wei Y, Jin Z, Zhang H, Piao S, Lu J, Bai L: The Transient Receptor Potential Channel, Vanilloid 5, Induces Chondrocyte Apoptosis via Ca2+ CaMKII-Dependent MAPK and Akt/ mTOR Pathways in a Rat Osteoarthritis Model. Cell Physiol Biochem 2018;51(5):23092323.

30 Zhao L, Ye J, Wu GT, Peng XJ, Xia PF, Ren Y. Gentiopicroside prevents interleukin-1 beta induced inflammation response in rat articular chondrocyte. J Ethnopharmacol 2015;172:100-7.

31 Jia N, Chu W, Li Y, Ding L, Duan J, Cui J, Cao S, Zhao C, Wu Y, Wen A: Iridoid glycosides from the flowers of Gentiana macrophylla Pall. ameliorate collagen-induced arthritis in rats. J Ethnopharmacol 2016;189:1-9.

$32 \mathrm{Lu} \mathrm{CH}$, Chung $\mathrm{CH}$, Lee $\mathrm{CH}$, Hsieh $\mathrm{CH}$, Hung YJ, Lin FH, Tsao $\mathrm{CH}$, Hsieh PS, Chien WC: Combination COX-2 inhibitor and metformin attenuate rate of joint replacement in osteoarthritis with diabetes: A nationwide, retrospective, matched-cohort study in Taiwan. PLoS One 2018;13:e0191242.

33 Martel-Pelletier J, Pelletier JP, Fahmi H: Cyclooxygenase-2 and prostaglandins in articular tissues. Semin Arthritis Rheum 2003;33:155-167.

34 Ansari MY, Haqqi TM: Interleukin-1beta induced Stress Granules Sequester COX-2 mRNA and Regulates its Stability and Translation in Human OA Chondrocytes. Sci Rep 2016;6:27611.

35 Kontny E, Rudnicka W, Kowalczewski J, Marcinkiewicz J, Maslinski W: Selective inhibition of cyclooxygenase 2-generated prostaglandin E2 synthesis in rheumatoid arthritis synoviocytes by taurine chloramine. Arthritis Rheum 2003;48:1551-1555.

36 Lowe GN, Fu YH, McDougall S, Polendo R, Williams A, Benya PD, Hahn TJ: Effects of prostaglandins on deoxyribonucleic acid and aggrecan synthesis in the RCJ 3.1C5.18 chondrocyte cell line: role of second messengers. Endocrinology 1996;137:2208-2216.

37 Amin AR, Attur M, Patel RN, Thakker GD, Marshall PJ, Rediske J, Stuchin SA, Patel IR, Abramson SB: Superinduction of cyclooxygenase-2 activity in human osteoarthritisaffected cartilage. Influence of nitric oxide. J Clin Invest 1997;99:1231-1237. 
38 DiBattista JA, Martel-Pelletier J, Cloutier JM, Pelletier JP: Modulation of glucocorticoid receptor expression in human articular chondrocytes by cAMP and prostaglandins. J Rheumatol Suppl 1991;27:102-105.

45 Tchetina EV, Kobayashi M, Yasuda T, Meijers T, Pidoux I, Poole AR: Chondrocyte

39 Sato T, Konomi K, Yamasaki S, Aratani S, Tsuchimochi K, Yokouchi M, Masuko-Hongo K, Yagishita N, Nakamura H, Komiya S, Beppu M, Aoki H, Nishioka K, Nakajima T: Comparative analysis of gene expression profiles in intact and damaged regions of human osteoarthritic cartilage. Arthritis Rheum 2006;54:808-817.

40 Barter MJ, Bui C, Young DA: Epigenetic mechanisms in cartilage and osteoarthritis: DNA methylation, histone modifications and microRNAs. Osteoarthritis Cartilage 2012;20:339349.

41 Yamamoto K, Okano H, Miyagawa W, Visse R, Shitomi Y, Santamaria S, Dudhia J, Troeberg L, Strickland DK, Hirohata S, Nagase H: MMP-13 is constitutively produced in human chondrocytes and co-endocytosed with ADAMTS-5 and TIMP-3 by the endocytic receptor LRP1. Matrix Biol 2016;56:57-73.

42 Pendas AM, Balbin M, Llano E, Jimenez MG, Lopez-Otin C: Structural analysis and promoter characterization of the human collagenase-3 gene (MMP13). Genomics 1997;40:222-233.

43 Billinghurst RC, Dahlberg L, lonescu M, Reiner A, Bourne R, Rorabeck C, Mitchell P, Hambor J, Diekmann O, Tschesche H, Chen J, Van Wart H, Poole AR: Enhanced cleavage of type II collagen by collagenases in osteoarthritic articular cartilage. J Clin Invest 1997;99:1534-1545.

44 Mitchell PG, Magna HA, Reeves LM, Lopresti-Morrow LL, Yocum SA, Rosner PJ, Geoghegan KF, Hambor JE: Cloning, expression, and type II collagenolytic activity of matrix metalloproteinase-13 from human osteoarthritic cartilage. J Clin Invest 1996;97:761-768. hypertrophy can be induced by a cryptic sequence of type II collagen and is accompanied by the induction of MMP-13 and collagenase activity: implications for development and arthritis. Matrix Biol 2007;26:247-258. 
54446 Borzi RM, Olivotto E, Pagani S, Vitellozzi R, Neri S, Battistelli M, Falcieri E, Facchini A, 545 Flamigni F, Penzo M, Platano D, Santi S, Facchini A, Marcu KB: Matrix metalloproteinase 13 loss associated with impaired extracellular matrix remodeling disrupts chondrocyte differentiation by concerted effects on multiple regulatory factors. Arthritis Rheum 2010;62:2370-2381.

47 Wu CW, Tchetina EV, Mwale F, Hasty K, Pidoux I, Reiner A, Chen J, Van Wart HE, Poole AR: Proteolysis involving matrix metalloproteinase 13 (collagenase-3) is required for chondrocyte differentiation that is associated with matrix mineralization. J Bone Miner Res 2002;17:639-651.

55348 Tewari P, Roy R, Mishra S, Mandal P, Yadav A, Chaudhari BP, Chaturvedi RK, Dwivedi PD, 554 Tripathi A, Das M: Benzanthrone induced immunotoxicity via oxidative stress and 555

49 Jeon J, Kang LJ, Lee KM, Cho C, Song EK, Kim W, Park TJ, Yang S: 3'-Sialyllactose protects against osteoarthritic development by facilitating cartilage homeostasis. J Cell Mol Med 2018;22:57-66.

50 Gardai SJ, Xiao YQ, Dickinson M, Nick JA, Voelker DR, Greene KE, Henson PM: By binding SIRPalpha or calreticulin/CD91, lung collectins act as dual function surveillance molecules

56251 Rokutanda S, Fujita T, Kanatani N, Yoshida CA, Komori H, Liu W, Mizuno A, Komori T: Akt 563 regulates skeletal development through GSK3, mTOR, and FoxOs. Dev Biol 2009;328:78564 93.

56552 Martin JA, Martini A, Molinari A, Morgan W, Ramalingam W, Buckwalter JA, McKinley TO: 566 Mitochondrial electron transport and glycolysis are coupled in articular cartilage. Osteoarthritis Cartilage 2012;20:323-329. 
A

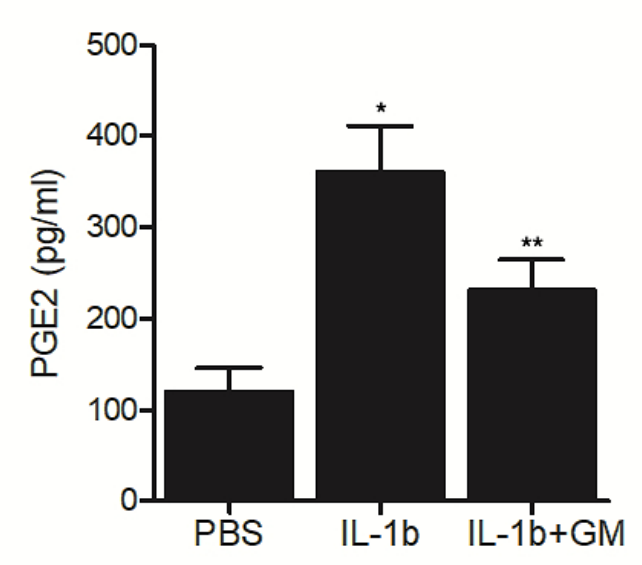

B

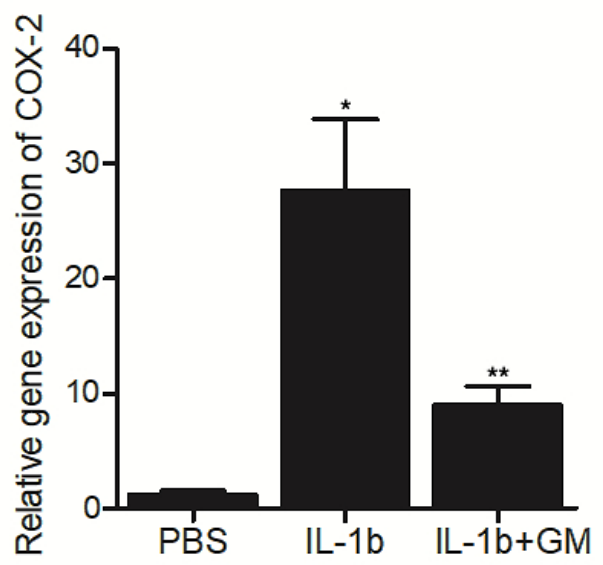

Fig.1

Expression of PGE2 and COX-2 mRNAs elevated by the IL-1 $\beta$ treatment was inhibited by the $\mathrm{GM}$ treatment $\left(\mathrm{N}=3 ;\right.$; $\mathrm{P}$ value $<0.05$ vs. PBS group; ${ }^{* *} \mathrm{P}$ value $<0.05$ vs. IL- $1 \beta$ group; one-way ANOVA)

A: ELISA assay indicated that PGE2 production was increased in primary rat chondrocytes treated with IL-1 $\beta$, while GM treatment inhibited the increased PGE2 production in IL-1 $\beta$ group;

B: Real time PRC indicated that the relative expression of COX2 mRNA was increased in primary rat chondrocyte s treated with IL-1 $1 \beta$, while GM treatment inhibited the increased PGE2 production in IL-1 $\beta$ group. 
A

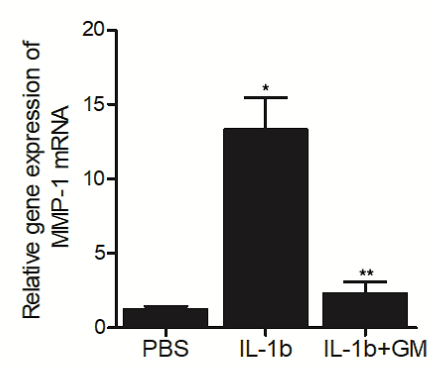

B

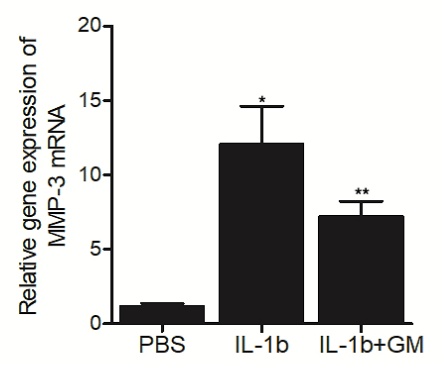

C

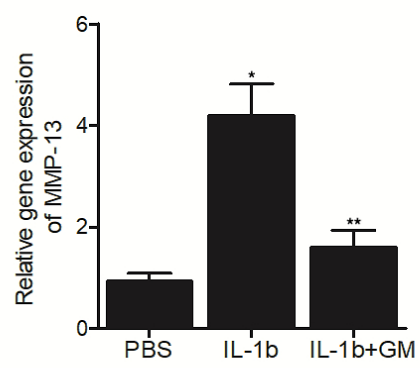

Fig.2

The expression of MMPs elevated by the IL-1 $\beta$ treatment was inhibited by the GM treatment (N-3; * $\mathrm{P}$ value $<0.05$ vs. PBS group; ${ }^{* *} \mathrm{P}$ value $<0.05$ vs. IL-1 $\beta$ group; one-way ANOVA) $A$ : Real time PRC indicated that the relative expression of MMP-1 mRNA was increased in primary rat chondrocyte s treated with IL-1 $\beta$, while GM treatment inhibited the increased MMP-1 mRNA level in IL-1 $\beta$ group;

$B$ : Real time PRC indicated that the relative expression of MMP-3 mRNA was increased in primary rat chondrocyte s treated with IL-1 $1 \beta$, while GM treatment inhibited the increased MMP-3 mRNA level in IL-1 $1 \beta$ group;

C: Real time PRC indicated that the relative expression of MMP-13 mRNA was increased in primary rat chondrocyte $s$ treated with IL-1 $\beta$, while GM treatment inhibited the increased MMP-13 mRNA level in IL-1 $\beta$ group. 

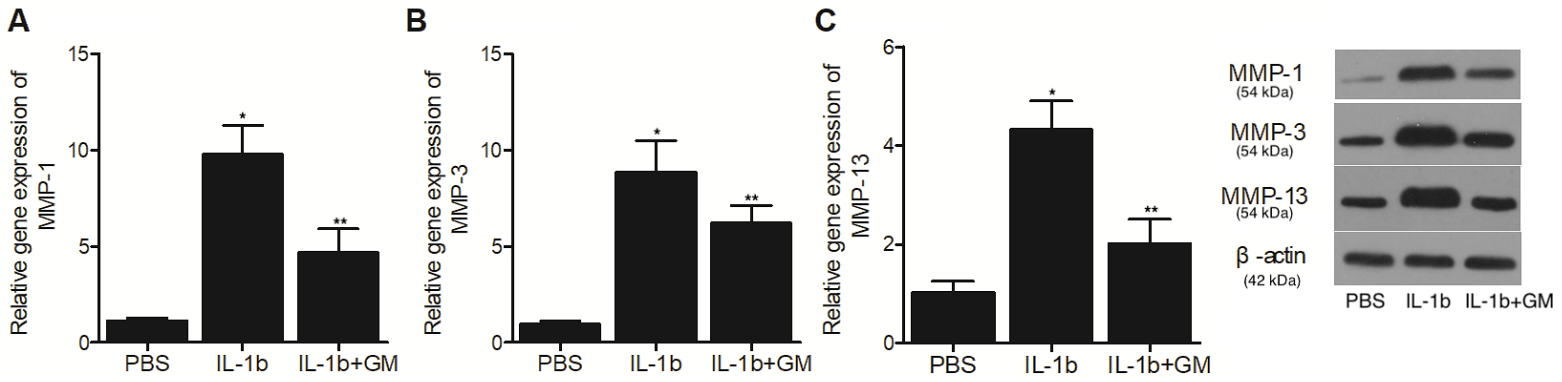

Fig.3

Protein expression of MMPs elevated by the IL-1 $\beta$ treatment was inhibited by the GM treatment $\left(\mathrm{N}=3\right.$; ${ }^{*} \mathrm{P}$ value $<0.05$ vs. PBS group; ${ }^{* *} \mathrm{P}$ value $<0.05$ vs. IL-1 $\beta$ group; one-way ANOVA)

A: Western blot assay indicated that the protein level of MMP-1 was up-regulated in primary rat chondrocyte s treated with IL-1 $\beta$, while GM treatment suppressed the up-regulation of MMP-1 protein in IL-1 $\beta$ group;

$\mathrm{B}$ : Western blot assay indicated that the protein level of MMP-3 was up-regulated in primary rat chondrocyte s treated with IL-1 $\beta$, while GM treatment suppressed the up-regulation of MMP-3 protein in IL-1 $\beta$ group;

C: Western blot assay indicated that the protein level of MMP-13 was up-regulated in primary rat chondrocyte s treated with IL-1 $\beta$, while GM treatment suppressed the upregulation of MMP-13 protein in IL-1 $\beta$ group. 


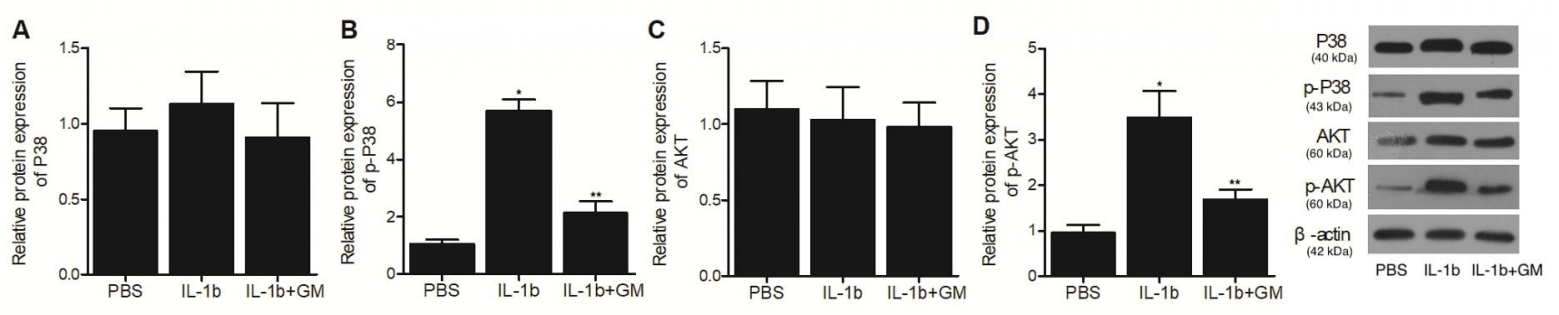

Fig.4

The protein production of $p-\mathrm{P} 38$ and $p-A K T$ elevated by the IL-1 $\beta$ treatment was inhibited by the $\mathrm{GM}$ treatment $\left(\mathrm{N}=3 ;\right.$; $\mathrm{P}$ value $<0.05$ vs. PBS group; ${ }^{* *} \mathrm{P}$ value $<0.05$ vs. IL-1 $\beta$ group; one-way ANOVA)

A: Western blot assay indicated that the protein levels of P38 remained stable among different primary rat chondrocyte groups;

$\mathrm{B}$ : Western blot assay indicated that the up-regulated protein level of p-P38 in primary rat chondrocytes treated with IL-1 $\beta$ was evidently reduced by the administration of GM;

C: Western blot assay indicated that the protein levels of AKT remained stable among different primary rat chondrocyte groups;

$D$ : Western blot assay indicated that the up-regulated protein level of p-AKT in primary rat chondrocytes treated with IL-1 $\beta$ was evidently reduced by the administration of GM. 

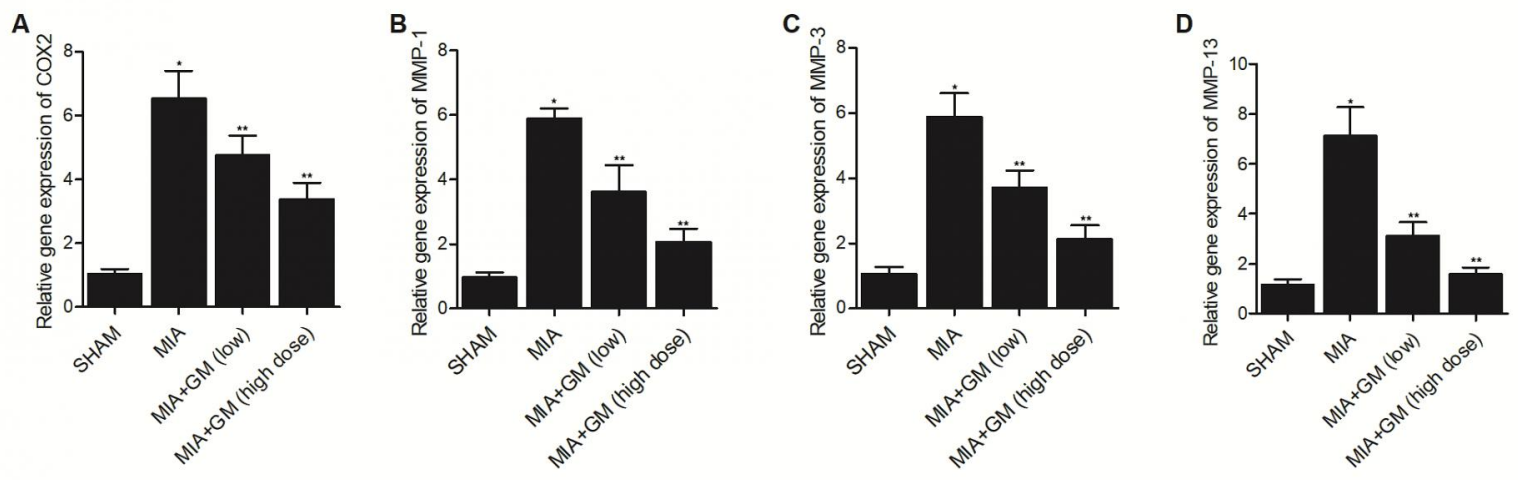

Fig.5

GM treatment inhibited the gene expression of COX2 and MMPs up-regulated in OA rats $\left(\mathrm{N}=3\right.$; * $\mathrm{P}$ value $<0.05$ vs. sham group; ${ }^{* *} \mathrm{P}$ value $<0.05$ vs. MIA group; one-way ANOVA) A: Real time PCR indicated that the relative gene expression of COX2 was significantly increased in MIA group, while being partly restored in MIA+GM group in a dose-dependent manner;

B: Real time PCR indicated that the relative gene expression of MMP-1 was significantly increased in MIA group, while being partly restored in MIA+GM group in a dose-dependent manner;

C: Real time PCR indicated that the relative gene expression of MMP-3 was significantly increased in MIA group, while being partly restored in MIA+GM group in a dose-dependent manner;

D: Real time PCR indicated that the relative gene expression of MMP-13 was significantly increased in MIA group, while being partly restored in MIA+GM group in a dose-dependent manner. 


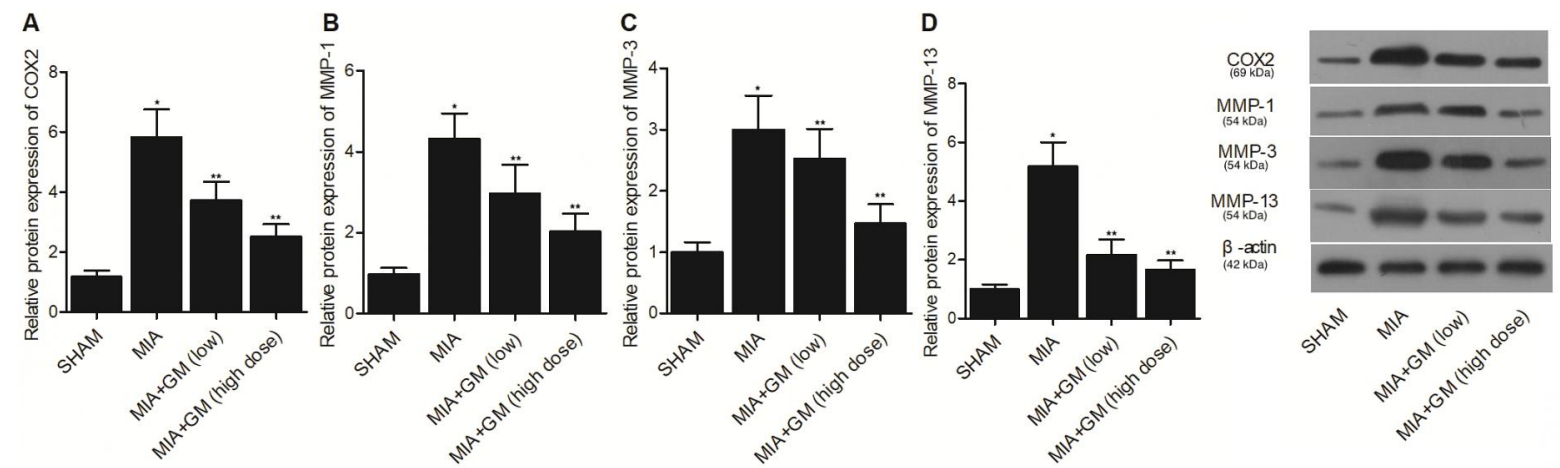

Fig.6

GM treatment inhibited the protein production of $\mathrm{COX} 2$ and MMPs up-regulated in OA rats $(\mathrm{N}=3$; * $\mathrm{P}$ value $<0.05$ vs. sham group; ** $\mathrm{P}$ value $<0.05$ vs. MIA group; one-way ANOVA) $A$ : Western blot assay indicated that the relative protein expression of COX2 was significantly increased in MIA group, while being partly restored in MIA+GM group in a dosedependent manner;

$\mathrm{B}$ : Western blot assay indicated that the relative protein expression of MMP-1 was significantly increased in MIA group, while being partly restored in MIA+GM group in a dosedependent manner;

C: Western blot assay indicated that the relative protein expression of MMP-3 was significantly increased in MIA group, while being partly restored in MIA+GM group in a dosedependent manner;

D: Western blot assay indicated that the relative protein expression of MMP-13 was significantly increased in MIA group, while being partly restored in MIA+GM group in a dosedependent manner. 


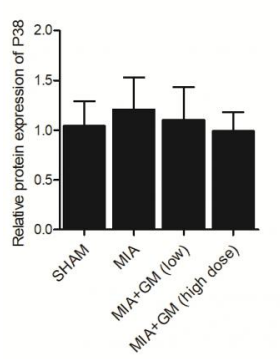

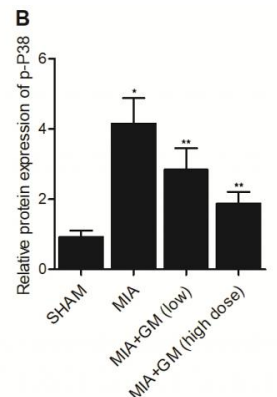
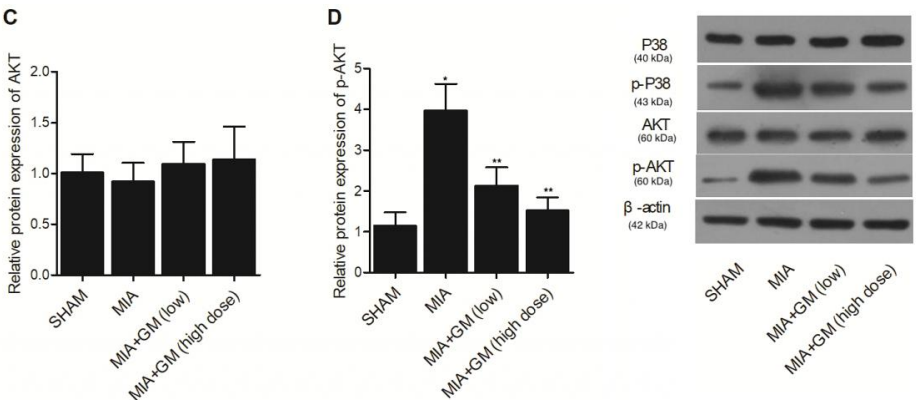

Fig.7

The Western blot assay showed that the GM treatment inhibited the p-P38 and p-AKT production which were up-regulated in $\mathrm{OA}$ rats $\left(\mathrm{N}=3 ;{ }^{*} \mathrm{P}\right.$ value $<0.05 \mathrm{vs}$. sham group; ${ }^{* *} \mathrm{P}$ value $<0.05$ vs. MIA group; one-way ANOVA)

$\mathrm{A}$ : Western blot assay indicated that the relative protein expression of $\mathrm{P} 38$ remained stable among different rat groups;

$\mathrm{B}$ : Western blot assay indicated that the relative protein expression of $\mathrm{p}-\mathrm{P} 38$ was evidently promoted in MIA group, and GM treatment partly restored the p-P38 up-regulation in MIA+GM group in a dose-dependent manner .

C: Western blot assay indicated that the relative protein expression of AKT remained stable among different rat groups;

D: Western blot assay indicated that the relative protein expression of p-AKT was evidently promoted in MIA group, and GM treatment partly restored the p-AKT up-regulation in MIA+GM group in a dose-dependent manner. 


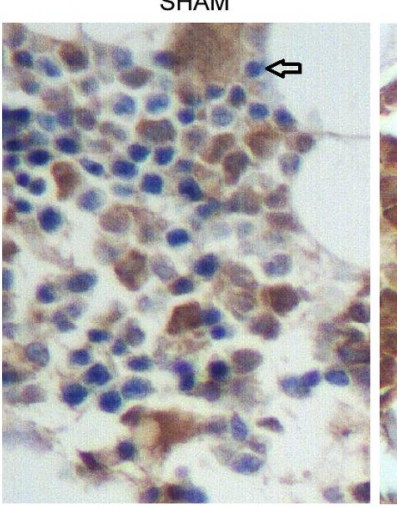

B

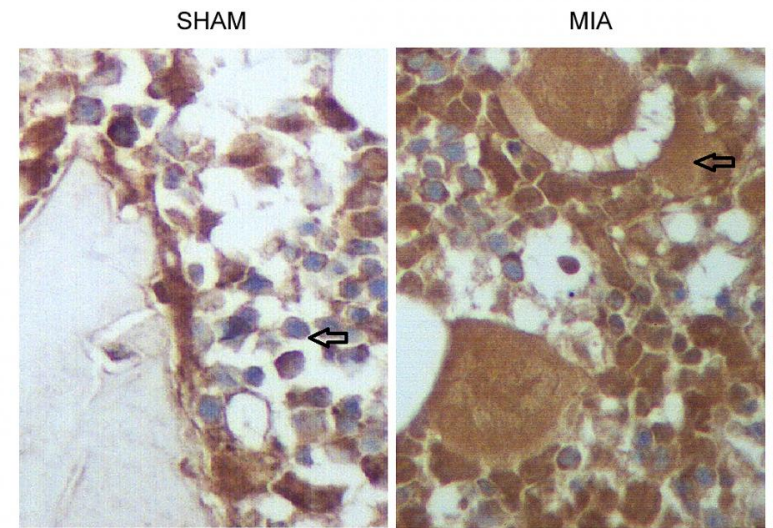

MIA

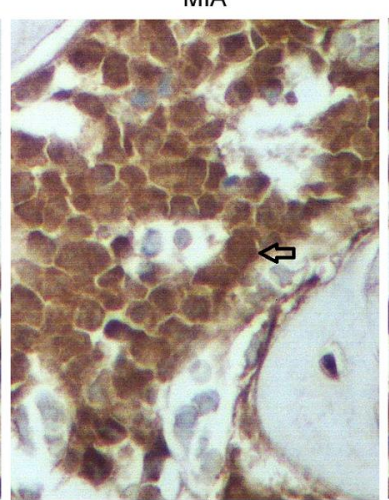

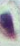

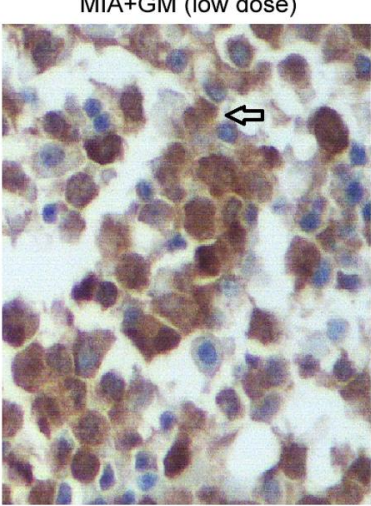

$M I A+G M($ low dose)

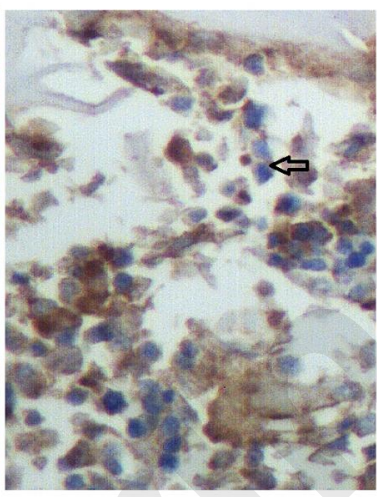

MIA+GM (high dose)

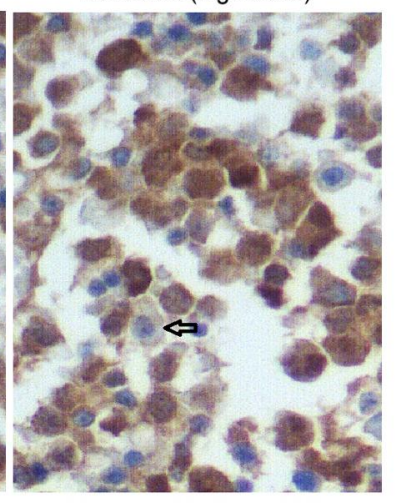

MIA+GM (high dose)

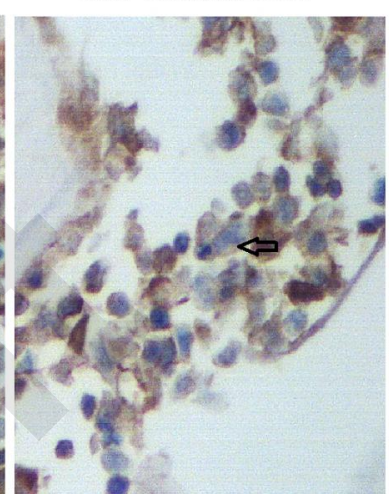

Fig.8 IHC assay showed that GM treatment inhibited the p-P38 and p-AKT production which were up-regulated in OA rats (Scale bar: $50 \mu \mathrm{m}$ ) A: IHC assay demonstrated inhibitory effect of GM upon the up-regulated expression of p-P38 in the MIA rats treated with different doses of GM; B: IHC assay demonstrated inhibitory effect of GM upon the upregulated expression of $\mathrm{p}-\mathrm{AKT}$ in the MIA rats treated with different doses of GM. 